\title{
Protée
}

\section{Le messie de la Mancha. Imaginaire et fin de la chevalerie dés orientée}

\section{Anne Élaine Cliche}

Volume 27, numéro 3, 1999

L’imaginaire de la fin

URI : https://id.erudit.org/iderudit/030569ar

DOI : https://doi.org/10.7202/030569ar

Aller au sommaire du numéro

\section{Éditeur(s)}

Département des arts et lettres - Université du Québec à Chicoutimi

ISSN

0300-3523 (imprimé)

1708-2307 (numérique)

Découvrir la revue

Citer cet article

Cliche, A. É. (1999). Le messie de la Mancha. Imaginaire et fin de la chevalerie dés orientée. Protée, 27(3), 29-44. https://doi.org/10.7202/030569ar
Résumé de l'article

L'auteur aborde avec Don Quichotte une analyse de l'imaginaire au sens de la psychanalyse. L'imaginaire de la " pureté de sang " propre à l'Espagne du Siècle d'or permet de ressaisir la dimension terrifiante et mortelle de ce registre dont le personnage de Cervantès devient le révélateur. C'est à la fonction du regard que le Chevalier de la Triste Figure nous convie pour rouvrir la captation imaginaire sur ce qui la fonde : le signifiant. Don Quichotte, comme les prophètes et le Messie de la tradition juive n'annonce pas la fin du monde, il dit l'actualité de la fin pour qui a oublié la fonction du regard, la place étrangère et sans image d'où la vérité d'une parole énigmatique et décentrée peut sortir. 


\title{
LE MESSIE DE LA MANCHA IMAGINAIRE ET FIN DE LA CHEVALERIE DÉSO RIENTÉE
}

ANNE ÉLAINE CLICHE

\begin{abstract}
Alors Iahvé envoya contre le peuple les serpents brûlants et ils mordirent le peuple: beaucoup moururent du peuple d'Israël. [...] Moïse intercéda pour le peuple et Iahvé dit à Moïse: «Fais-toi un serpent brûlant et mets-le sur une hampe: quiconque aura été mordu et le verra, il vivra!» Moïse fit donc un serpent d'airain et le plaça sur la hampe. Or si l'un des serpents mordait un homme et que celui-ci regardait vers le serpent d'airain, il vivait!
\end{abstract}

$\mathrm{Nb}$ XXI, $6-91$

Le messianisme appartient d'abord à l'histoire juive, aux deux versants de l'histoire juive que sont le judaïsme et l'antisémitisme. Pour la tradition juive, le messie - de l'hébreu machiah, "oint" - est le sauveur et rédempteur qui apparaîtra à la fin des temps. Quant au messianisme, il comprend à la fois l'idée d'une restauration et d'une utopie. Voilà pour les généralités. La logique de la figure est plus complexe et participe d'une interprétation plusieurs fois reprise, déplacée, élargie, ressaisie surtout après le "coup christique", selon l'expression de Daniel Sibony, pour s'affirmer au cours des siècles et dans les divers cadres d'élaboration de la tradition. La littérature du Second Temple, contemporaine du surgissement d'un messie nommé Jésus et de plusieurs autres que l'histoire a oubliés; la pensée talmudique qui se construit entre le deuxième et le cinquième siècle de notre ère; la mystique et la kabbale; les divers mouvements religieux; toutes ces versions du judaïsme déploient les composantes de la figure du messie de manières diverses. Il y a pourtant un «trait unaire» en ces variations, et qui relève de la généalogie; généalogie dont la Bible se fait en quelque sorte l'archive, le matériau historique. Les recensements nombreux qui scandent la Torah, les mariages mixtes, pérégrinations, incestes, prostitutions qui forment le matériau premier des Écritures, constituent d'une certaine manière l'exposé détaillé et humain des origines et de la filiation «impure» du messie.

À la question «D'où vient le Messie?», la raison rabbinique élabore d'ailleurs une réponse étonnante qui résonne avec celle de la Bible et ne repose que sur la 
logique de la lettre hébraique. On se souviendra que chaque lettre de l'hébreu correspond à un nombre, ce qui permet les jeux d'esprit les plus inattendus dont la causalité provient de correspondances anagrammatiques ou simplement mathématiques.

Ainsi, dans le troisième livre du Pentateuque (Torah), Moïse combat une invasion de serpents en obligeant ceux qui ont été mordus à regarder un serpent d'airain: "Celui qui le regarde vit». Les rabbins nous apprennent que le serpent - en hébreu $\mathrm{NaHaCH}$ - a la même valeur que le messie - en hébreu MaCHIaH -; entendez que l'addition des lettres de chacun de ces mots produit la même somme: 358. Cette équivalence numérique, suivant les règles talmudiques, induit donc une équivalence des «valeurs». En effet, nous disent les Sages, le serpent est du côté de la vérité; il ne ment pas et engendre en chacun la remise en cause de ce qu'il croyait vrai. De tout temps, enfin, sa présence est facteur de la connaissance qui donne à l'homme accès à la responsabilité de ses actes. La Torah transcende ainsi les dualismes primaires et noue d'emblée la vérité à la figure la plus corrosive et la plus sinueuse qui soit.

Les principes talmudiques nous invitent à procéder résolument par associations; associons donc:

De nombreuses sociétés humaines, d'une manière obsessionnelle, s'acharnent à tuer ce serpent qui pourrait les faire évoluer. Faute de le « regarder " [...] elles se condamnent à mourir. ${ }^{2}$

D'où vient le messie? De la matière même, littérale, de l'animal déclaré le plus impur, le plus immonde. Il me semblait important de rappeler cet aspect de la question, histoire de la reprendre par le bout qui la rattache non seulement à l'imaginaire du salut, mais à la «loi du genre» biblique et talmudique: loi littéraire, historique et tordue.

\section{IMAGINAIRE DE LA MANCHA}

La peur maladive de la mancha, de la tare [ou tache] d'une lointaine histoire juive, passe par son apogée de 1580, en gros, à 1650-1660. Elle contribue à figer la société espagnole dans un refus collectif de mobilité. [...] Protégé par son insignifiance, le petit peuple des villes et des campagnes est passionnément antisémite. Il s'est fabriqué en termes de vieille chrétienté, une noblesse en forme de contre-noblesse. Pierre Chaunu ${ }^{3}$

L'anecdote du serpent d'airain de Moïse nous rappelle par ailleurs à une fonction singulière $d u$ regard. En quoi le fait de regarder l'image fabriquée du serpent peut-elle redonner vie au sujet contaminé? Cette question me donnera, si l'on veut bien me suivre à la vitesse qui s'impose ici, le cadre d'une relecture du roman de Cervantès; relecture qui, dans les limites de cet article, ne saurait prétendre à une contribution sérieuse aux études cervantines, et trouve plutôt dans l'écriture de Don Quichotte l'occasion de mettre en lumière, ne serait-ce qu'un peu, l'actualisation - et l'actualité - d'un véritable «traitement» de l'imaginaire de la fin.

«En un lugar de la Mancha, de cuyo nombre no quiero acordarme, no ha mucho tiempo que vivia un hidalgo...» 4 ainsi commence la musique du Quichotte: rythmée, andante, errante et très orientée. Les traductions sont des facteurs de surdité considérables, ce n'est un secret pour personne. Et le français, comme les autres langues étrangères à celle de Cervantès, nous installe sans préavis, au commencement du Livre, dans une Espagne d'emblée géographique, celle de l'atlas, ouvert là, sous mes yeux, exhibant sa constellation de petits villages dont la Manche, contrée de la Nouvelle Castille, est peuplée. La polyphonie est pourtant convoquée comiquement, pour l'oreille de tout hispanophone, aux premiers mots de L'Ingénieux hidalgo: «En un lieu [lugar] de la tache [mancha]... et village de la Manche». Voilà bien où commence cette histoire au moins double, et où s'annonce l'ingéniosité en question. La géographie, du coup, passe au registre métaphysique dont nous ne perdrons plus ni la résonance ni l'envoûtante étrangeté, doublure sinon revers d'une physique imposante et brutale, celle du corps même de don Quichotte, entravé, surchargé, traversant le paysage crissant d'une Espagne toute pragmatique et hantée. La mancha est justement ce qui occupe tous les esprits et peut 
retourner votre vie comme un gant; elle est à elle seule le nom de votre finitude la plus envisageable, la plus probable, arrêt de mort signé avec votre sang, et signifiant premier d'un imaginaire collectif de la fin qui a saisi l'Espagne, du 15e au 19e siècle, jusqu'à la rendre insensée, anachronique, mystique et funèbre.

Toute l'histoire de l'Inquisition espagnole peut s'écrire à partir de ce mot magique; hantise généalogique de la tache, tare biologique avant la lettre, la mancha coule dans le sang d'une génération à l'autre sans s'effacer, maculant au contraire ascendants et descendants qu'aucune lustration baptismale ne saurait purifier. L'Espagne des statuts de limpieza de sangre est travaillée par l'obsession de la contamination ${ }^{5}$. L'imaginaire de la pureté va donc se constituer un corps spéculaire unifié en cette figure du lignage immaculé, sans conversion, et surtout sans histoire, dans tous les sens du terme. Corps mythique dont chacun doit se saisir, qui en brûlant ses papiers de famille, qui en détruisant ses archives ou en changeant de nom pour se refaire une généalogie fictive sans trou, ni blanc, ni tache: ni juif ni maure.

L'Inquisition passe, tout entière, corps et biens, au service des passions, des rancœurs, des intérêts et des représentations de la masse vieille chrétienne. À la hauteur des années 1580, le torrent est devenu un grand fleuve en crue, qui n'épargne plus rien. Les statuts de pureté de sang ont tout envahi, tout contaminé. Il n'y a plus de secteur sain. L'obsession est générale. 6

La pureté se fantasme au nom de l'Église et de sa mission universelle dont les Espagnols se sentent directement investis. C'est précisément de cette image de l'Espagne, figée dans sa consistance eschatologique, acharnée dans sa prétention à l'Immaculée Conception dont l'instauration du dogme va d'ailleurs lancer le pays dès le début du XVIIe siècle dans une véritable croisade ${ }^{7}$, que va «sortir» - car c'est littéralement d'une sortie qu'il s'agit - l'écriture de Don Quichotte, entre 1600 et 1616, année de la mort de Cervantès.

L'imaginaire messianique propre à l'Espagne catholique ne relève pas, on va le voir, de l'imagination, trait plutôt propre à caractériser don Quichotte, mais d'une structuration du discours fascinée par l'image unifiante du corps, celui d'une Nation purifiée, absolue et sainte, élue d'une élection nouvelle, usurpée par un catholicisme étatique, «en proie», pourrait-on dire, à l'Incarnation. En 1492, l'Espagne, par ses rois catholiques, a fait un pacte divin.

1492. L'Espagne achève son unité, expulse ses juifs, et Dieu la récompense aussitôt pour avoir purifier son sol par la découverte des Amériques. D'ailleurs, Ferdinand le Catholique souligne de nombreuses fois le lien entre l'expulsion des juifs et le don de

Dieu. Qu'importe si le prix de l'or est le sang indien! Tous les succès de l'Espagne sont les fruits d'un zèle religieux exceptionnel qui n'a pas laissé insensible la Providence divine. C'est pourquoi se forge très vite dans la mentalité espagnole la croyance en une mission: propager la vraie foi dans l'univers tout entier. ${ }^{8}$

Ce pacte sera maintenu dans l'Espagne des Habsbourg, autrement resserré sous Philippe II et Philippe III, rois du Siècle d'Or dont la cour est établie respectivement à Madrid et à Valladolid ${ }^{9}$. Le messianisme espagnol se constitue comme une appropriation de l'élection divine, usurpant une «position" métaphysique transmise antérieurement à un peuple qu'il renie, expulse, mais dont il reste "occupé». En fait, dès que l'on revendique l'élection divine, on se heurte à une monolithique antériorité obligeant celui qui s'identifie aux signes de cette élection à de subtiles nuances. L'usurpation de l'élection procède toujours du même aveuglement. Le principe consiste à voir dans l'élection un privilège, une participation exclusive au corps divin, une puissance supplémentaire qui vous met du côté de Dieu, sans entrevoir le poids de la dette que recèle l'alliance, dette qui n'est en l'occurrence même plus pensable. L'identification imaginaire au peuple élu est imaginaire en ce qu'elle fonctionne par projection d'une image chrétienne ou païenne - pure et divine du messianisme sur l'histoire des Hébreux. Identification aux signes construits et non à l'impératif qui en est la condition. Car l'élection - au sens de la Torah - n'est ni un pouvoir ni un mérite, 
encore moins un privilège ou une communion; elle est une acceptation de la Loi, une condition et un devoir. Condition humaine dont le messianisme, dans sa version juive première, est la figure d'achèvement éthique et d'accomplissement historique. Le messianisme chrétien, on le sait, a divinisé la figure et, de ce fait, a "expulsé» la dette au profit du corps glorieux. C'est à ce corps de gloire que s'agrippe l'Espagne de Cervantès, dont le picaro se fait, bien entendu, l'inversion concertée, et que don Quichotte, pour sa part, ramène obstinément et vaillamment dans le champ du regard ${ }^{10}$.

\section{LE RETOUR DE L'EXPULSÉ}

Le moi est à l'origine un moi corporel, il n'est pas seulement un être de surface, mais il est lui-même la projection d'une surface. Freud ${ }^{11}$

L'imaginaire, au sens de la psychanalyse (Lacan), n'est pas sans entretenir certains rapports avec ce corps glorieux qui est, en quelque sorte, la transposition religieuse de l' «avènement» de l'image corporelle constitutive du sujet parlant. Si cette image constitutive est le moment de saisie du corps comme tout, et risque d'occuper tout l'espace des relations ultérieures au monde, elle n'en demeure pas moins tributaire du regard qui la soutient et la "troue», dans la mesure où ce regard en est expulsé, excentré et ouvre dans l'image cette place invisible d'où le «je» s'éprouve avec ce corps mais au-delà. La capture de l'image opère d'abord par ce croisement des regards que constitue la rencontre avec l'image du corps autre comme «mien». Le regard n'est pas l'œil mais ce qui, au champ de l'Autre - hors champ - me fait d'abord, au commencement du monde, objet visible, regardé.

De sorte que, le voyant étant pris dans cela qu'il voit, c'est encore lui-même qu'il voit: il y a un narcissisme fondamental de toute vision; et que, pour la même raison, la vision qu'il exerce, il la subit aussi de la part des choses [...] - ce qui est le sens second et plus profond du narcissisme: non pas voir dans le dehors, comme les autres le voient, le contour d'un corps qu'on habite, mais surtout être vu par lui, exister en lui, émigrer en lui, être séduit, capté, aliéné par le fantôme, de sorte que voyant et visible se réciproquent et qu'on ne sait plus qui voit et qui est vu. 12

L'imaginaire. Qu'est-ce à dire au juste? Avant toute considération psychologique ou morale, l'imaginaire se définit comme la modalité première de l'image. Qu'est-ce que l'image, demandera-t-on? Une forme visible, consistante dans la mesure où le regard peut la saisir, la reconnaitre, la reprendre, la reproduire. La science optique nous rappelle que pour qu'une image ait une certaine consistance, il faut qu'à chaque point de l'objet corresponde un point de l'image et que tous les rayons issus d'un point se recoupent quelque part en un point univoque. Principe du plan de projection dont la fonction de saisie de l'image comme image exige du regard qu'il en soit expulsé, hors champ. La psychanalyse a permis quant à elle de faire entendre que l'imaginaire est d'abord le corporel, non pas l'objet du biologiste mais l'image du corps. Expérience première et mythique de l'humain qui ne voit sa forme réalisée, totale, le mirage de lui-même, que hors de lui-même, et ne se ressaisit comme sujet que depuis cette extériorité interne, dans la mesure où la captation spatiale de l'image passe par celle du corps regardant. Le corps qui regarde est aussi regardé. Et c'est cette réciprocité spéculaire qui constitue à la fois l'imaginaire complétude de la forme corporelle et sa trouée de l'intérieur d'où «je» parle et regarde. L'imaginaire est ce registre de projection de l'un sur l'autre, dont le corps propre est en quelque sorte le paramètre inaugural. Projection de l'image, du corps "comme tout», sur l'écran du corps-moi et réciproquement, et sur l'écran du monde dont le langage, le discours, constitue en quelque sorte le matériau de tissage.

L'imaginaire procède donc d'un rapport au monde dont la modalité est de reconnaissance, d'identification, de ressemblance ou de dissemblance, registre de l'amour et de l'agressivité dont l'aliénation provient de sa composition essentiellement duelle qui méconnaît justement la béance du «je» ex-centré, et joue de la captation unifiante, du leurre de la complétude. 
À ce titre, l'imaginaire n'est donc ni le contenu ni le contenant de ce que j'imagine mais un attachement indépassable à l'ordre du visible, une saisie du corps, le mien, dans et par l'image de mon semblable, qui commande ensuite mon rapport à toute extériorité. Il n'y a de moi constitué - l'expérience de l'inconscient en offre une mise à l'épreuve répétée - que de l'image de l'autre. Dans la mesure où il s'agit d'un registre, d'une modalité de la rencontre avec la réalité et avec l'autre, l'imaginaire est toujours imaginaire de la fin. La forme unifiée et la jubilation qu'elle procure, puisqu'elle signe l'entrée dans la puissance de la coordination - l'enfant s'éprouvant "démembré» avant de s'apparaître «tout un »-, constitue la limite du corps propre et, avec elle, l'espace physique de son accomplissement. Imaginaire de la fin, en ce sens aussi que l'image, sa fixation, offre à voir la clôture du sujet, dans la mesure où le «je» qui participe de l'image mais n'y «apparaît» pas - parce qu'il se voit tel qu'en lui-même l'autre le change en image - s'y trouve aliéné. Le regard, place du Je, est d'emblée au champ de l'Autre, première altérité que l'imaginaire ne reconnaît que sur le mode d'une agressivité qui marque toute relation à l'autre. En fait, la psychanalyse nous apprend que la parole vient au sujet depuis l'assomption de cette image du corps unifiée, c'est dire l'incomplétude qui est la sienne. L'image de l'autre comme moi-même, cette dualité qu'impose la projection d'un plan sur l'autre, propre à toute image, introduit une tension, un jeu-je, une béance que seule la parole comme alliance et séparation peut soutenir.

Le registre de l'imaginaire quant à lui ne propose qu'un affrontement duel dont la seule résolution possible serait la suppression réelle de l'image de l'autre: issue de la psychose, singulière ou collective. L'imaginaire cherche la fin de l'incomplétude éprouvée, ressentie, la résolution de cette tension entre moi et l'autre, étranger comme moi-même. Dans les sursauts de violence qu'il met en scène, c'est la place du regard qu'il oublie, oblitère; cette place ouverte d'où le «je» expulsé ne cesse de revenir pour dire ce qui ne saurait se voir, l'imaginaire la méconnaît et la nie. L'imaginaire est le registre particulier de cette négation de l'incomplétude et de l'inadéquation. Cette consistance unifiée qui cherche, pourrait-on dire, sa pureté de contour, «donne la formule la plus générale de la folie, celle qui git entre les murs des asiles, comme celle qui assourdit la terre de son bruit et de sa fureur" ${ }^{13}$.

Dans l'appareil d'unification qu'invente l'Espagne de Don Quichotte, on reconnaît bien, pour reprendre le terme de Lacan, «ce noud de servitude imaginaire» ${ }^{14}$. Il m'a semblé que le roman de Cervantès, devenu en quelque sorte le Grand Livre - la Bible - de l'Espagne, énonçait la décision de reconnaitre ce nœud et de le trancher; autant dire de provoquer, par la force d'une écriture particulière, le retour de cette place excentrée, du regard expulsé. La folie de l'Espagne à l'apogée de l'Inquisition, au cœur du Siècle d'Or, donne, je pense, la mesure à une autre folie, celle de don Quichotte, étrange et décidée, fermement orientée vers un but lui aussi étrange, énigmatique, et dont l'aliénation singulière, comme fabriquée à l'envers de l'imaginaire, a de quoi nous sortir, encore maintenant, de nos captations les plus tenaces.

\section{CHÂTEAUX EN EsPagne}

[...] comme à notre aventurier tout ce qu'il pensait, voyait ou s'imaginait lui semblait être fait et se passer de la même façon que ce qu'il avait lu, tout aussitôt qu'il vit la taverne, il se représenta que c'était un château [...]. En ces entrefaites, il advint qu'un porcher qui ramassait parmi les chaumes un troupeau de cochons (car, sauf excuse ils s'appellent ainsi) sonna un cornet au son duquel ils se ramassent, et à l'instant se représenta à don Quichotte ce qu'il désirait, qui était que quelque nain donnât le signal de sa venue. (I, II, 75-76)

La folie de l'Espagne inquisitoriale semble devenue un lieu commun pour les historiens ${ }^{15}$; folie qui pourrait se résumer dans la Reconquête, au XVe siècle, du territoire musulman vécue comme un remembrement, une recollection du corps démembré fondée sur l'unité religieuse, et de plus en plus soucieuse de souder ses morceaux avec ce que Henry 
Méchoulan appelle «le sang des autres ou l'honneur de Dieu». Folie qui est aussi l'effet de la Conquête d'un Nouveau Monde apparemment reçu en don, cadeau de Dieu qui rivalise sur le mode imaginaire avec les Tables de la Loi au Sinaï. L'imaginaire de ce don est en effet palpable puisqu'il n'est que la projection de l'image d'un «bien» donné et reçu, sur l'écran d'une scène biblique qui permet précisément de diviniser l'Espagne, de la sacraliser comme corps glorieux. Le pacte divin demeure imaginaire non parce que les Rois espagnols s'imaginent simplement être destinataires des faveurs de Dieu mais, plus profondément et plus structuralement, parce que ce Nouveau Monde qu'ils reçoivent en don est perçu comme une "récompense», un privilège mérité, et permet d'effacer la dette qu'impose quant à elle la Loi de Moïse ${ }^{16}$. Si la rivalité avec le peuple élu assumait l'enjeu réel de cette appropriation, elle devrait en passer par la reconnaissance du sens singulier que prend le don advenu au Sinaï. Don de la Loi qui n'a rien d'une récompense et dont le premier impératif consiste justement à interdire la fabrication des images, autant dire qu'elle prévient les puissances du visible pour rappeler le regard et la parole à la mémoire de l'alliance - qui n'est pas la commémoration d'une communion avec et dans le corps. Le don de la Loi impose un lien non fusionnel qui ordonne la «séparation", la coupure d'avec le tout, l'arrachement à la captation de l'image.

La décadence de l'Espagne - déjà pressentie et amorcée en plein éblouissement du Siècle d'Or provient de cette consistance imaginaire d'un pouvoir puisant ses figures dans la Bible hébraïque sans en lire l'interprétation qui compose pourtant l'essentiel du récit. Là réside, d'ailleurs, toute l'ironie, le paradoxe, sinon la confusion de l'affaire. L'Espagne catholique ne fait pas qu'expulser ses juifs (et plus tard en 1609, entre les deux livres du Don Quichotte, ses maures), ce qui est déjà un symptôme suffisant. Elle s'approprie l'histoire des Hébreux (élection, messianisme et sainteté) sans en assumer la condition qui est assujettissement à la Loi, et que le juif - c'est précisément ce qui le distingue des autres et constitue son élection - a le devoir de rappeler au reste du monde. En retour de cette identification imaginaire, l'Espagne se voue à une piété exemplaire.

Il ne faudrait pas croire cependant, souligne Méchoulan, que le zèle excessif du clergé espagnol se manifeste exclusivement en oraisons. Le maintien de la pureté de la foi est aussi son affaire, affaire que partage la nation tout entière. Aussi laïcs et religieux célèbrentils ensemble les exploits du Saint-Office, autre don de Dieu. 17

L'Espagne glorieuse n'a plus dès lors qu'à s'occuper de sa pureté et de sa purification, qui l'occupe d'ailleurs au point de l'arracher au Temps et à l'Histoire, bref au symbolique qui est un ordre, celui de la parole, du dire, du signifiant, de l'agencement non spécularisable du monde et de sa loi ${ }^{18}$.

L'Espagnol, rivé à sa solitude, paye le prix de la pureté de son sang. Il manque la révolution cartésienne, les grands bouleversements intellectuels de l'Europe pour avoir refusé de «feuilleter le grand Livre du monde» autrement qu'avec la pointe de son épée. 19

Mais la purification, l'effacement de la tache, semble devoir être infinie, inachevable. Depuis 1492, il n'y a plus de juifs en Espagne, l'alternative partir ou se convertir a aboli leur présence "visible». On sait que plusieurs de ceux qui restèrent acceptèrent sincèrement la conversion, et que d'autres, nombreux, «judaïsèrent» en secret tout en affectant une piété chrétienne, condition de leur survie. Ce sont ces convertis d'apparence que l'on appelle les marranes (de marrano: porc). Dès lors, tout un chacun peut être soupçonné de crypto-judaïsme, la conversion étant devenue le masque constamment soulevé-redéposé sur le visage espagnol. L'excès de pureté devient ainsi une véritable contamination qui finira par engendrer chez certains la croyance tenace que tout Espagnol est un marrane, un juif masqué.

Et c'est du lieu de cette mancha obsédante, proliférante que va s'effectuer la première «sortie» de don Quichotte; première sortie précédée, comme on va le voir, par une entreprise soutenue de nomination, causalité première des aventures à venir. Cette 
première sortie sera d'ailleurs fort brève et consistera essentiellement en sacre de notre chevalier devant l'auge (pila ${ }^{20}$ ) baptismale d'une taverne renommée "château " par le valeureux hidalgo.

D'où vient don Quichotte, et de quelle ingéniosité traite son histoire? Le roman, il va sans dire et tout le monde l'a dit, est énigmatique et peut être lu sur le mode théologique, cabalistique, historique, sociologique, psychanalytique ${ }^{21}$. Chacune de ces lectures semble y trouver les assises de son épistémè et chacune circule dans le dédale des questions et étrangetés sans pouvoir honnêtement ni conclure ni trancher ni fermer le livre. Quel sens peuvent bien avoir ces sorties successives et ces déambulations finalement restreintes dans l'espace du territoire? Que signifient cette comédie de chevalerie errante et ces dialogues philosophiques entre don Quichotte et Sancho, dignes de ceux qui ont cours entre un maître et son disciple? Je ne vais pas à mon tour tenter de répondre à ces questions somme toute impertinentes. Le roman avoue son but et le répète à satiété: en finir avec l'infâme et médiocre roman de chevalerie. Cette décision donne d'ailleurs l'occasion d'un prologue qui vient en quelque sorte justifier sur le mode humoristique l'absence supposée et soulignée d'érudition du livre. L'ami alter ego venu soutenir l'auteur padrastro (beau-père) de l'histoire que l'on va lire n'hésite pas à dire:

Et puisque votre composition ne tend qu'à ruiner l'autorité et le crédit que les livres de chevalerie ont acquis au monde parmi le commun peuple, il n'est pas besoin que vous alliez mendier sentences de philosophes, conseils de la Sainte Écriture, fables de poètes, oraisons de rhétoriciens, ni miracles de saints. (I, 57)

Prenons-le aux mots. En effet, l'enseignement du Don Quichotte ne relève pas de l'érudition mais d'un véritable travail de la matière, philosophique, théologique, poétique, rhétorique et miraculeuse. Le lecteur "desocupado", oisif, désoccupé, à qui s'adresse le texte n'a qu'à recevoir ce récit de seconde, voire de troisième ou quatrième main, et à le lire pour voir s'abattre «la machine mal fondée de ces livres chevaleresques" (I, 58). Il s'agit, nous dit-on encore, de faire en sorte que le mélancolique soit ému à rire, que le rieur le soit encore plus, et que le sage donne à cette histoire quelques louanges.

Le problème, rétorque-t-on souvent, est qu'on ne voit plus tout à fait ce qu'il y a de drôle à combattre des moulins à vent, à crever des outres de vin, à se faire sacrer chevalier devant une auge à cochon. N'y at-il donc là qu'humour de quiproquos? De quoi rit-on au juste quand nous rions avec le Quichotte? Certains ont dit que ramener le texte à son contexte constituait une limitation impardonnable et que l'universalité du premier roman moderne tient justement dans le fait qu'il échappe à son contexte pour traverser l'Histoire et la géographie ${ }^{22}$. Procédons à l'inverse de ce lieu commun, pour voir si l'extrême enracinement dans le temps et le lieu n'est pas justement la condition de l'universalité concrète de l'énigme que soulève ce livre. L'ingéniosité ici n'est ni la nôtre ni celle du livre - du moins ne s'affiche-t-elle pas d'emblée - mais celle de l'hidalgo don Quichotte de la Mancha. Son art est en tout cas mystérieux, lui qui se caractérise par des actes manqués à répétition et une disposition «systématique» à l'illusion. C'est d'ailleurs dans ce «système» qu'il faudrait sans doute voir l'ingéniosité, système que je voudrais ici déplacer quelque peu en marge des lectures reçues.

Certes, il y a imitation et accomplissement des livres dans le monde, avec l'inadéquation que cela suppose et qui, en effet, pousse à rire. Certes, le personnage est mû par un «idéal» de justice dans un monde déchu qui ne sait plus reconnaître les valeurs fondamentales, etc. Mais, franchement. Relisons encore une fois cette histoire tordue. Le système semble encore nous échapper et paraît parfois aller justement à l'encontre de cet entendement pourtant soutenable. Don Quichotte accomplit de véritables carnages, profane des enterrements, empire les supplices des victimes... et s'avance tel un endeuillé inguérissable, imposant sa foi à coups d'épée et sa Dulcinée à coups de prières et d'aveux forcés. Dans le second volume, le chevalier rencontre ses propres lecteurs et semble se faire la dupe de leurs machineries à illusions grossières et programmées. Il y a là comme 
une tristesse qui vous gagne. Que ce passe-t-il au juste? Les guérisseurs de sa folie, à la fin, triomphent. Don Quichotte guérit et meurt dans son lit. Quelque chose, là, échappe, et pourtant nous sentons que la vérité nous attend ailleurs, qu'elle n'est pas simplement éludée, niée, indifférente. Ingénieux ce don Quichotte, lui qui semble toujours faire l'envers de ce qu'il prétend faire? Inversons donc nous-mêmes le problème. Cette apparente inadéquation au réel ne serait-elle pas l'ingéniosité par excellence? Et ce deuil de la chevalerie perdue, égarée, n'est-il pas aussi ce qui dans le livre s'affirme avec le plus de clarté et de lumière?

Ingenio, en espagnol c'est le génie, l'esprit, comme le mot d'esprit est une figure de l'esprit. L'esprit, dit Baltasar Gracian (Jésuite espagnol du XVIIe siècle), est «la cause efficiente» de l'acuité (agudeza) qui est, pourrait-on dire, le registre de la vérité porté non à l'évidence du visible mais à la fonction du regard. Autrement dit, l'esprit est un art, et l'art de l'esprit n'est possible que là où il s'incarne: dans l'acuité d'un regard qui retourne l'image comme un gant ${ }^{23}$. La figure de rhétorique incarnée n'est pas simple trope, mais corps parlant et regardant. La figura - ainsi, d'ailleurs, appelle-t-on don Quichotte à plusieurs reprises, lui qui sera renommé par Sancho el Caballero de la Triste Figura - est le lieu de l'incernable esprit. Cette triste figure dit bien la pauvreté et la maigreur de la forme en question. Figure qui, comme le dit bien Sancho, ne nécessite aucune reproduction et parle d'elle-même. À don Quichotte qui, ravi de cette nomination - qu'il croit inspirée par le «sage» chargé d'écrire l'histoire de ses exploits -, désire peindre sa triste figure en guise de nom sur son écu, Sancho rétorque:

Monsieur, il n'est aucun besoin d'employer du temps et de l'argent à faire faire cette figure, il suffit que vous découvriez la vôtre et montriez le visage à ceux qui vous regarderont: car sans plus, et sans autre image ni écu, ils vous appelleront celui de la Triste Figure. (I, XIX, 212)

Les châteaux de don Quichotte - châteaux réels, auberges, hangars, etc. - sont tous des châteaux en
Espagne au sens où non seulement ils apparaissent comme des chimères, mais parce qu'ils sont les échafaudages du projet certainement chimérique du livre tout entier: en finir avec la chevalerie. L'expression "château en Espagne» nous arrive directement de la chevalerie selon laquelle il était courant de donner en fief, en concession, un château au chevalier reconnu. Mais ce château donné à titre de tenure noble, le chevalier devait d'abord l'attaquer et le prendre. En finir avec la chevalerie ne se réduira pas à la dénigrer, mais reviendra plutôt à l'imiter, à s'en faire le «miroir" non pour la reproduire, on l'aura compris, mais pour révéler la faille sur laquelle toute image repose, pour redonner à entendre ce qu'elle a elle-même oublié. Car la chevalerie visée est aussi celle de l'Espagne et son idéal de chevalier chrétien livré aux sortilèges de l'image d'un monde plein et pur.

Le chemin de la Croix tracé par l'épée, telle était la voie simple et droite qui s'offrait depuis la Reconquête à l'espagnole. À quelques exceptions près, aucune réflexion n'est venue troubler la transparence du projet mystique et militaire. Vivant pour

l'Espagne, l'élu de Dieu, le héros du XVIe siècle sentait palpiter en lui l'histoire dont il était l'instrument. Eniuré par la conscience permanente d'avoir été choisi par la divine Providence, il percevait la réalité quotidienne comme sacrée. 24

«Flambeau et miroir de toute la chevalerie errante», don Quichotte de la mancha apparaît de plus en plus comme un regard porté sur cette chevalerie dés-orientée, dé-sémitisée. Sa folie est d'ailleurs extraordinairement limitée à ce domaine: hors de la chevalerie point de folie mais au contraire, tous les personnages en témoignent, haute sagesse. Don Quichotte veut ressusciter ce que le livre désire achever. Principe de la comédie ici à l'œuvre. Mais cette dialectique permet aussi de pousser à l'extrême l'imaginaire de la fin, de le cadrer pour le donner à entendre comme vecteur de catastrophe. De là, le deuil déborde le personnage et travaille le livre, vient prendre de court ce qui depuis toujours l'empêchait: une réalité figée dans sa gloire, et sacrée. D'où l'on verra que l'imagination peut devenir une arme terrible et nécessaire contre les puissances de 
l'imaginaire. On ne s'étonnera pas, dès lors, que le cornet du porcher rassemblant ses cochons-puercos (qui, sauf excuse, s'appellent aussi marranos) marque l'entrée de don Quichotte sur la scène de sa première aventure.

\section{EN FINIR AVEC L'ORIENT:}

\section{LA BIBLIOTHĖQUE EST EN FEU}

À la première page du roman, après le prologue et les poèmes dédicatoires, un portrait de l'hidalgo s'ébauche à partir des maigres et rares composantes de son alimentation ${ }^{25}$. Le lecteur espagnol tombe dès la deuxième phrase sur un os. Dans la liste des aliments énumérés qui composent les repas de la semaine, nous lisons que les samedis, l'hidalgo mange duelos y quebrantos, littéralement deuils (ou peines) et brisements de coeur (ou afflictions, délabrements). Étrange repas qui ne semble pourtant s'enchaîner à aucune autre métaphore puisque le reste est composé d'une marmite contenant "plus de bœuf que de mouton, un saupiquet la plupart du temps les soirs, des lentilles le vendredi et quelques pigeonneaux de surcroît le dimanche». Même l'édition espagnole demeure hésitante à «traduire» ces deuils et brisures: «On ne sait pas concrètement, écrit Luis Casanovas Marqués, quelle est cette nourriture: peut-être était-ce les abats de l'animal (tête, pieds, foie) ou bien des œufs au lard ${ }^{26}$. Jean Cassou affirme quant à lui: «Les commentateurs et traducteurs ont disputé sans fin de ces duelos y quebrantos, deuils et brisures, locution qui, en Castille et dans la Manche, signifiait des œufs au lard, ainsi que l'avait traduit notre César Oudin, et que l'ont établi les plus récents cervantistes [...]»27. Létonnant, n'est-ce pas, est que l'on considère nous avoir renseignés sur la chose. Il y a là pourtant apparence de mot d'esprit, expression idiomatique, tournure singulière dont certains ont tenté de retrouver l'origine en l'associant à l'obligation pour le marrane de manger du lard. Le samedi, en Espagne, est à l'époque un jour de surveillance redoublée puisqu'il s'agit de traquer le juif «persistant» qui, en ce jour béni et saint, risque de se révéler. Manger des œufs au lard, un samedi de surcroît, est sans doute en effet pour un juif un acte qui change le jour saint en un jour de deuil et de brisement de cœur. En fait, et le juif et le maure sont concernés par cet endeuillement que provoque le fait de manger du porc, viande interdite par les deux religions et prisée par l'Espagne catholique qui en fait le signe de l'appartenance au corps unifié.

À partir de ces petits détails qui, il est vrai, sont nombreux dans le roman, on a pu avancer que Cervantès était juif ou marrane ${ }^{28}$. L'idée est fascinante et, en effet, risque de fasciner la lecture au point de l'arrimer à la lettre d'une interprétation par avance cryptée, cabalistique ou autre. Mais on doit surtout rappeler que la langue espagnole a conservé de nombreuses traces des deux cultures sémites, arabe et juive, qui ont participé intimement à son développement. Don Quichotte le rappelle lui-même à Sancho lors d'une conversation qui le force à passer par les détours de l'étymologie (II, LXVII, 555) 29. Toutes ces remarques n'empêchent pas que l'on puisse aussi se contenter de ce qui est écrit: l'hidalgo encore sans nom mange régulièrement des deuils et brisures. Le reste de l'histoire, d'ailleurs, ne contredit en rien cette affirmation claire et limpide; et puisque le livre commence là, nous devons aussi le lire à partir de là. On peut rappeler encore qu'après cette brève énumération qui précède de quelques lignes à peine l'entrée en scène du nom, nous ne verrons plus guère le personnage manger sinon pour indiquer à quel point cela lui est difficile, voire indifférent quand ce n'est contraire à son éthique. Chaque fois, en tout cas, la scène est significative. «Pour moi Sancho, je suis né pour mourir d'une mort continuelle, et toi pour mourir en mangeant» (II, LIX, 487). La dimension mystique du personnage est en tout cas prégnante, et le fameux «Je meurs de ne pas mourir» de la sainte d'Avila pourrait être prononcé par l'Espagne tout entière ${ }^{30}$.

Après la description des repas arrive celle des vêtements et du corps. Puis vient le nom. L'entreprise de nomination, ici, est double. L'auteur hésite entre quelques noms parce que, dit-il, «on veut dire qu'il avait le surnom de Quixada ou Quesada (car en ceci il 
y a encore quelque différend entre les auteurs), encore que par conjectures vraisemblables on pense qu'il s'appelait Quixana; mais cela importe peu à notre conte: il suffit qu'en la narration d'icelui on ne sorte un seul point de la vérité» (I, I, 68). C'est donc aussi le nom de l'auteur qui se trouve, là, pointé, laissant ouverte la place d'où le texte nous arrive. Quant au personnage, il se renomme délibérément, opérant de là un premier déplacement.

Cette double nomination construit aussi, dans l'organisation de ces toutes premières pages, le cadre à une critique brève et incisive des romans de chevalerie dont on dit que l'hidalgo est "enfrasco", c'est-à-dire quelque chose comme "embouteillé», «enflaconné», autant dire imbibé, la lecture lui ayant en quelque sorte embarrassé le goulot. Décidé, donc, à devenir lui-même chevalier, il commence par renommer son roussin Rossin-antes (roussin-avant), pour que le nom témoigne de la métamorphose. Quant au nom don Quichotte, qui vient au chevalier après huit jours passés à y penser, il a donné l'occasion comme on sait de multiples interprétations ${ }^{31}$ dont celle qui emprunte à la langue hébraïque n'est pas la moins intéressante puisque $Q(e) c h(e) t$ en hébreu signifie justement vérité et nous renvoie directement à Em(e)t, nom de l'auteur substitut fictif du livre que nous lisons et qui prend la relève au chapitre IX de la première partie, Cide Hamete Benengeli ${ }^{32}$. Don Quichotte joindra à son nom de chevalier le lieu de son pays, la Mancha, "par où, à son avis, il déclarait fort clairement sa race et sa patrie, et l'honorait beaucoup en prenant le nom d'icelle» (I, I, 72). Prenons donc ce fil de la vérité qui, loin d'être ténu, se tisse de manière serrée au reste des aventures.

Les romans de chevalerie qu'a lus l'hidalgo Quixada-Quesada-Quixana-Quisade, et avec lui la société espagnole, sont présentés par deux pseudocitations qui en constituent si l'on peut dire le pastiche inaugural.

La raison de la déraison qui se fait à ma raison de telle sorte affaiblit ma raison qu'avec raison je me plains de votre beauté. Les beaux cieux qui de votre divinité divinement vous fortifient avec les étoiles et vous rendent méritante du mérite que mérite votre grandeur.
Ces deux phrases, qui apparaissent là en italiques, concernent comme par hasard «la raison de la déraison» et les mérites que mérite celle qui a la grandeur divine. Nous sommes en plein dans la question contemporaine à l'écriture du livre, et cette raison de la déraison ressemble aussi, si l'on veut bien un moment s'attacher au contexte, à la folie de l'Espagne encore imbibée des valeurs de la chevalerie, qui veut justement mériter les mérites que le ciel lui accorde et qui, pour ce faire, prône un bien, une justice et une pureté absolue, absolument vieillechrétienne. Il s'agira donc pour le livre de poursuivre ce travail de sape à travers les déplacements de ce don Quichotte messianique cherchant à défaire les torts envers et contre tous pour le compte d'un nom divin qui n'a d'ailleurs de divin que le nom: Dulcinée du Toboso.

Au retour de la première sortie qui se fait sur un âne et semble parodier l'entrée du Messie en terre sainte $(\mathrm{I}, \mathrm{V}, 96)^{33}$, une seconde critique des livres s'effectue par la visite du barbier et du curé en la bibliothèque du chevalier. Le bûcher, ici, s'orchestre à partir d'une sélection à la fois arbitraire et concertée, et rappelle évidemment les bûchers alors nombreux qui occupent l'Espagne. Brûler, en cette circonstance précise de la fiction, se présente comme une cure, une démarche de guérison sinon de rédemption, ordonnée par le curé et le barbier, dont on verra d'ailleurs qu'elle a peu d'effet sur la suite de l'histoire. L'important dans cet acte de la déraison familière, outre qu'il permet un véritable commentaire, voire une apologie des livres que possède l'hidalgo, réside sans doute dans le fait qu'il sera, après coup, attribué au «Maure enchanté», ennemi juré de don Quichotte, et qui s'avère à partir de là détenir le fil de l'histoire et celui, décentré, de la vérité. Autrement dit, ce qu'on brûle sur la place publique - et qui est l'Orient sémitique dont l'Espagne est non seulement tributaire mais pétrie - devient ici le maître du jeu. L'inversion est donc définitivement installée et rien ne pourra la défaire sauf à surmultiplier les simulacres d' "enchantements". À cette chevalerie de mauvais goût, que l'Inquisition elle-même avait mise à l'Index 
sans trop de succès, le livre s'associe apparemment en mimant jusqu'à son cadre d'énonciation ${ }^{34}$; mais au premier tour de l'esprit ici au travail, c'est une chevalerie «désorientée» qui semble plutôt visée, une chevalerie qui cherche à en finir avec l'Orient qui la hante et envers lequel elle ne se veut reconnaître ni dette ni deuil. Cervantès met donc en scène un chevalier explicitement orienté, se croyant «enchanté» par le Maure, et repartant à la première occasion, accompagné cette fois d'un «vieux chrétien» avec lequel le dialogue et l'enseignement pourront s'effectuer. Cette deuxième sortie sera d'ailleurs brutalement interrompue, comme si le livre que nous étions en train de lire était à son tour flambé, déchiré, puis retrouvé par hasard dans les tiroirs secrets d'une juiverie qui nous donnera cette fois le véritable cadre de cette aventure de la raison. Le roman de don Quichotte se donne donc à lire comme un «retour de l'expulsé», l'auteur se démultipliant au service d'une vérité toute particulière: celle de l'imaginaire de la fin.

\section{L'ENCHANTEUR POURRISSANT ${ }^{35}$}

Le cousin et Sancho écoutaient avec une grande attention les propos de don Quichotte, qu'il proférait, comme s'il les eût arrachés de ses entrailles avec une douleur extrême. Ils le supplièrent de leur donner à entendre ce qu'il disait et de leur dire ce qu'il avait vu dans cet enfer [la caverne de

Montésinos]. [...]

- Et les personnes enchantées, dit le cousin, mangent-elles?

- Elles ne mangent pas, repartit don Quichotte, ni ne font les gros excréments [...].

- Et les enchantés dorment-ils par hasard? dit Sancho.

- Non, certes, répliqua don Quichotte [...].

- Oh! que ce proverbe, dit alors Sancho, convient bien ici: dismoi qui tu hantes et je te dirai qui tu es.

(II, XXIII, 203-204)

Cervantès affirme être le parrain d'un livre dont il ne possède, au départ, que les premiers chapitres. Au chapitre IX de la première partie nous apprenons que la suite a été retrouvée dans la juiverie de Tolède, en une version rédigée en arabe par Cide Hamete
Benengeli, et que l'«auteur» se fait traduire en castillan qu'il retranscrit pour nous. Ce cadre énonciatif n'est, à partir de là, jamais oublié et devient au contraire de plus en plus envahissant, laissant place plus d'une fois aux commentaires de Cide Hamete rédigés dans les marges. Le récit que fait don Quichotte de sa descente dans le monde enchanté de la caverne de Montésinos gardera quant à lui le statut de texte apocryphe, Cide Hamete affirmant douter franchement de la vérité de cette histoire qui «va trop loin des lois de la raison» (II, XXIV, 208). La transcription de cette aventure nous est donc tout de même livrée, puisque recueillie par les transcripteurs précédents, mais sans que l'on puisse affirmer, contrairement au reste des aventures, qu'elle soit vraie ou fausse. Cette descente en enfer apparaît donc, comiquement, comme un trou dans le texte qui en est aussi la mise en abyme, puisque tout le livre est donné pour apocryphe. Mais le récit semble ici vouloir creuser davantage sa fonction, au moment où don Quichotte descend dans le monde des enterrés vivants, monde de l'enchantement généralisé qui n'en finit pas de hanter l'esprit de la chevalerie.

En fait, don Quichotte semble être celui pour qui l'enchantement arrive. Et l'Espagne fictive qu'il traverse paraît n'avoir d'autre occupation que d'alimenter cet enchantement funeste, leurre qui, dans le second livre, prend des proportions encore plus insistantes, manifestes, comme exposées de force au regard «désoccupé» du lecteur; proportions presque grossières, douloureuses en somme pour qui s'est fait complice de l'extraordinaire lucidité du personnage en toutes matières, exceptée la chevalerie. Il s'agit, dirait-on, de nous extraire du mythe, de «désenchanter» l'Espagne et nous avec elle, car il n'est pas dit, comme le soutenait Artaud, que l'envoûtement ne soit pas aussi notre lot. Il y a dans l'Espagne de don Quichotte quelque chose de pourri, quelque chose qui pourrit mais ne meurt pas et continue obstinément de parler, de revenir. Le juif expulsé - dont on ne parle jamais directement dans le roman - est devenu cryptique, c'est-à-dire omniprésent, menaçant plus que tout autre étranger la 
généalogie chrétienne qui, en voulant purifier sa lignée, fait paradoxalement outrage aux origines même du Messie-Jésus; mais il ne s'agit pas de remonter si haut. Quant au Maure, devenu la figure sémitique encore nommable bien qu'indésirable et bientôt expulsée à son tour, il est le dernier représentant de l'Orient dans cette lutte à finir que lui livre toute la scène méditerranéenne. Mais voilà. Contre l'imaginaire mythique de la pureté éternelle, Cervantès restitue une logique d'engendrement dont la folie «systématique» de don Quichotte, folie travaillée par le retour dans l'image de ce qui a été jeté au dehors, est garante.

Il suffit de prendre une aventure parmi tant d'autres. Au chapitre XVII du premier volume, deux troupeaux de moutons et de brebis marchant l'un vers l'autre apparaissent aux yeux du chevalier sous la forme d'une confuse bataille entre deux armées dont l'une est celle de l'empereur Alifanfaron qui est un «furibond païen devenu amoureux de la fille de Pentapolin [chef de l'autre armée], qui est fort belle et fort gracieuse dame et qui est chrétienne». L'enjeu de la guerre est que "son père ne la veut bailler à ce roi païen, si premièrement il ne quitte la loi de son faux prophète Mahomet et ne se convertit à la sienne» (I, XVII, 197). Ce que voit don Quichotte est un réel dont la rencontre des moutons peut momentanément soutenir l'indicible question: celle d'une alliance sexuelle, disons d'un désir, fracassant l'interdit dogmatique. C'est le regard du chevalier qui provoque l'entrée de l'invisible Histoire dans l'histoire. Par ailleurs, le roman traite la figure mauresque à plusieurs reprises sur ce mode sexuel qui s'offre aussi comme la trouée dans l'image spéculaire unifiante. Trou de plus en plus narratif qui semble par endroits retourner l'histoire sur elle-même lorsqu'on assiste à ces récits de chrétiens vivant sous domination islamique, convertis de force et pratiquant en secret le culte catholique ${ }^{36}$. Inversion de l'histoire prêtant sa structure de fiction à la vérité qui ne saurait se dire en tant que telle.

Tous les actes fous que pose don Quichotte sont attribués au Maure enchanté, véritable corps cryptique enfermé dans la conscience hispanique. Or ces actes manqués apparaissent petit à petit comme des réussites agissant sur un autre plan, dans la doublure de l'histoire qui retourne à chaque scène la bande du texte. Le sémite est couché au fondement de la culture espagnole, et gît tel Durandard au fond de la caverne de Montesinos (II, XXIII) dont le récit se donne comme apocryphe, c'est-à-dire impur quant à son origine. Le roman ne lâche à aucun moment sa prise, la tirant vers les dessous de l'image, revers que compose l'imagination débridée de don Quichotte, imagination orientée, sémitisée à dessein, livrant la lecture à sa propre dis-simulation. Car dans cette Espagne des expulsions et des autodafés, la simulation est un principe d'existence et la dissimulation un vrai acte de foi. Cervantès place le lecteur sur cette tranche de l'image, sur sa coupe où il est constamment menacé de passer par le trou de l'imagination qui n'est que deuil, dette et doute travestis dans les espèces de la foi.

\section{LA TRANSSUBSTANTIATION: NOUS SOMMES TOUS DES MARRANES}

Je sais qui je suis, répondit don Quichotte, et sais que je peux être non seulement ceux que j'ai dits, mais aussi tous les douze pairs de France et tous les neuf preux de la Renommée. (I, V,97)

C'est là, bien sûr, que l'on rejoint l'universalité du problème. L'Espagne inquisitoriale pourrait bien être vue comme l'expression la plus poignante de la «servitude imaginaire» constitutive du sujet de la parole. Servitude devenue loi royale retombant sur les sujets comme la raison d'État. De là, si l'on peut dire, tout le monde s'arrange tant bien que mal. Et le marranisme, qui est en quelque sorte l'effet de cette servitude, constitue un phénomène assez remarquable d' «arrangement». La condition du marrane - nom méprisant par lequel on désigne le converti (musulman mais surtout juif) dont on juge que la conversion est feinte; nom qui recouvre les termes nouveau chrétien, crypto-juif ou crypto-musulman, converso, etc. -; la condition marrane relève en quelque sorte 
d'une doublure que l'on impute au sujet converti et qui finit par le définir réellement dans la mesure où la conversion forcée, même si parfois elle rejoint un acte de foi sincère, impose une métamorphose ostentatoire, toujours appelée à fournir la preuve de son authenticité. Le converti doit donc "afficher» sa foi nouvelle, la simuler en quelque sorte même si elle est réelle puisqu'elle participe d'un système de signes qui fonctionne comme dispositif visible derrière lequel on suppose aussitôt le secret. Autrement dit, la simulation, l'excès de signes revendiqué, engendre le principe d'une dissimulation - actualisée ou non qui construit, invente un sujet par essence masqué. L'intéressant dans la personnalité marrane est qu'elle se définit par une ambiguïté, une duplicité non pas seulement provisoire et circonstanciée mais «transmise» comme condition même de la parole constamment éprouvée dans son double registre de simulation et de dissimulation.

Dans la société inquisitoriale, le marrane ne peut vivre, et surviure, que s'il maîtrise l'art de paraître totalement le Même que l'Autre, tout en restant, en son existence la plus profonde, la plus intime, autre. 37

Mais celui qui demeure intimement juif tout en participant résolument à l'identité du groupe majoritaire développe au cours des générations une identité particulièrement clivée, même lorsque la déperdition du savoir sur la tradition cultivée en secret est devenue presque totale. En fait, le marrane se trouve confronté si l'on peut dire à un second «stade du miroir» dans la mesure où c'est l'image de l'autre qui détermine la sienne: identité d'un Moi saisie par l'identité de l'Autre, qui, par ce second tour, le constitue «comédien» du monde. Le marrane vit de manière aiguë la division intrinsèque au sujet humain, il en devient en quelque sorte le représentant, voué qu'il est à soutenir cette division parfois jusqu'à la folie, jusqu'à la mélancolie ${ }^{38}$.

Tout le roman de Cervantès, d'ailleurs, participe de cette logique, au point de permettre l'assomption du corps réel en l'espèce du leurre. Une scène célèbre nous donne d'ailleurs les matériaux de ce que j'essaie ici de dire. «Où l'on traite de l'héroïque et formidable bataille que don Quichotte livra à des outres de vin rouge» (I, XXXV). Le valeureux et très chrétien chevalier, se trouvant couché à l'auberge parmi les outres de vin, se voit en rêve attaqué par un géant. Se dressant sur ses pieds, à demi nu, il lance donc son épée dans ce corps illusoire, et transperce ainsi les outres qui se répandent. Don Quichotte affirme aux hôtes qui se précipitent pour l'arrêter qu'il vient de couper la tête à l'enchanteur dont on peut voir «le sang sortir du corps comme une fontaine». Le tavernier a fort bien entendu: "Quel sang et quelle fontaine, dis-tu, ennemi de Dieu et de ses saints?».

Il semble bien que nous ayons affaire en l'occurrence à une sorte de transsubstantiation, d'ailleurs opérée de tout temps par le chevalier. Transsubstantiation qui est, comme on le sait, l'objet central de la vraie foi et l'envers du visible. On peut en tout cas se rappeler que l'ostentation propre à la religion marrane bute en quelque sorte contre cet os du visible que constitue le sacrifice de la messe. Ce sacrifice - dont on sait que le peuple juif fut nommé responsable et auquel on l'oblige à "croire", à défaut de quoi, c'est à sa propre mise à mort qu'il est conviéa donné lieu à un dogme qui vise directement le monde de l'image et de la représentation. «Ceci est mon corps ceci est mon sang» constituent donc les mots clés d'un réel impossible à voir et pourtant avéré par la foi catholique, donné pour réel envers et contre toute "raison" 39 . Don Quichotte habite un monde en proie à la transsubstantiation, celle-ci n'opérant d'ailleurs, comme il se doit, que dans le champ limité à la foi qui en pose l'effectivité.

Dans cette logique du signe et du contre-signe, le porc joue un rôle essentiel. Interdit de consommation par les religions juive et musulmane refoulées et expulsées par l'Espagne, devenu le nom-insulte qui les désigne, le porc accède finalement, pour le chrétien en mal de preuve, au statut de «bouclier», d'écran protecteur, signe suprême de l'appartenance. Les proverbes de Sancho en sont d'ailleurs littéralement tissés, tel celui-ci qui revient à plusieurs reprises: «Là où il y a des chevilles, il n'y a pas toujours du lard " 40 . 
Que les personnages inquiétés par l'Inquisition - comme le morisque Ricote, chassé avec sa famille en 1609 et croisant don Quichotte alors qu'il revient incognito sur ses terres chercher son bien (II, LIV) - se promènent avec des os de jambon, a de quoi faire rire ceux, nombreux à l'époque, qui connaissent le code.

\section{LE PORC DE LA VÉRITÉ REFOULÉE}

Se contenant donc dans les étroites limites de la narration, quoiqu'il ait assez d'habileté, de capacité et d'intelligence pour traiter de l'univers, [Cid Hamete] demande qu'on ne rabaisse pas son travail, et qu'on lui donne des louanges, non pour ce qu'il a écrit, mais pour ce qu'il a laissé d'écrire. (II, XLIV, 360)

Qu'a donc «laissé d'écrire» - ou laissé ne pas s'écrire - l'auteur fictif de l'ingénieuse histoire que nous lisons ${ }^{41}$ ? Que n'a-t-il pas écrit dont on devrait le louanger? Il y a dans ce commentaire en marge comme un silence pointé, présenté à cet endroit sous les espèces d'un refus de digression et de bavardage; causalité qui vient enrober une proposition qu'il conviendrait sûrement de prendre, une fois de plus, à la lettre. La figure du porc serait en tout cas à suivre de près pour faire entendre ce qui, justement, ne saurait s'écrire. On se souvient de l'entrée décidée de don Quichotte dans la première aventure de l'histoire, à l'appel du porcher. Lisons donc maintenant la dernière, intitulée littéralement la "porcine aventure qui arriva à don Quichotte (II, LXVIII). On trouve, en effet, à la fin du second livre, un événement dernier que vivront le chevalier et son écuyer avant de rentrer au village natal. Installé à la belle étoile avec son fidèle Sancho, vaincu par le chevalier masqué qui n'était qu'un prétendu ami souhaitant sa guérison, et forcé de rentrer chez lui, don Quichotte au plus profond de sa mélancolie entend soudain un bruit sourd. La brutalité du surgissement n'a d'égale que son horreur: six cents pourceaux que l'on mène à la foire arrivent telle une "horde grognante» qui piétine sans ménagement le chevalier, l'écuyer, son grison et
Rossinante. Bouleversé par l'arrivée violente de ces «animaux immondes», don Quichotte ordonne à Sancho qui cherche son épée: «Laisse-les aller, l'ami; cet affront est la peine de mon péché. Le Ciel, par juste châtiment, veut qu'un chevalier errant qui s'est laissé vaincre soit mangé des chacals, piqué par des guêpes et foulé des pourceaux». Mais le "péché» de don Quichotte est-il bien celui qu'il pense? Cette aventure nocturne, précédée d'une conversation entre don Quichotte et Sancho sur la nécessité et l'urgence de "désenchanter" Dulcinée, ressemble justement à un désenchantement radical. Le péché n'est-il pas plutôt celui de la sainteté qui s'obstine à défaire les torts; péché de la chevalerie errante, égarée dans le miroir de sa gloire? Chassez le porc - maure, juif ou marrane - il revient au galop. Le Messie de la tache, ne serait-il pas celui qui vous impose de la regarder? Tache aveugle du regard qu'il importe de remettre dans le champ de l'image pour en trouer la pureté illusoire.

La rédemption que propose L'ingénieux hidalgo don Quichotte de la Manche n'a pas tout à fait le sens d'une lustration. Elle relève au contraire de l'exposition de la dette refoulée, de son retour comme châtiment pour qui ne sait pas reconnaître sa condition de divisé. Le messianisme de don Quichotte n'annonce pas la fin du monde, il dit l'actualité de la fin pour qui a oublié la fonction du regard, il dit la place étrangère et sans image d'où la vérité d'une parole énigmatique et décentrée peut sortir. Ce messianisme affirme le retour de l'immonde dans le monde qui cherche à en finir avec l'Autre. L'animal immonde, porc ou serpent, juif ou maure, doit donc être «regardé» par cette ouverture que le livre pratique dans le plan de projection.

Ce qui n'a pas été écrit n'est pas pour autant absent du livre; cette béance, ce blanc ouvert dans le texte explicitement désigné par l'auteur comme objet de louanges, condition de son art, apparaît à la fin comme le lieu même d'où nous le lisons. 


\section{N O TES}

1. Trad. d'É. Dhorme, La Bible. Ancien testament I, Paris, Gallimard, Bibliothèque de la Pléiade, 1956.

2. R. Cohen, Tora au présent, Paris, Buchet/Chastel, 1994, p. 22.

3. "La société en Castille au tournant du Siècle d'Or ", Revue d'histoire économique et sociale, $\mathrm{n}^{\circ} 45,1967, \mathrm{p} .159$.

4. «En un village de la Manche, du nom duquel je ne me veux souvenir, demeurait, il n'y a pas longtemps, un gentilhomme... ", trad. C. Oudin, revue par J. Cassou, Paris, Gallimard, coll. «Folio», 1949. Dans la suite du texte nous renverrons à cette édition, publiée en 2 vol. correspondant aux deux «parties » parues respectivement en 1605 (ce premier livre se divisait d'abord en quatre parties) et en 1615 (Cervantès redivise alors l'ensemble selon les deux livres), par une parenthèse indiquant, dans l'ordre, le volume, le chapitre et la page de la citation.

5. Les «statuts de pureté de sang» sont des clauses restrictives introduites dans les règlements régissant le fonctionnement des associations et des institutions publiques et privées. Le premier est adopté à Tolède en 1449. L'apparition de l'Inquisition en 1480 crée l'amalgame entre impureté de sang et hérésie et contraint les membres de cette société, extrêmement métissée depuis le Moyen Âge sous domination musulmane, à masquer leurs ascendances inavouables. Être vieux chrétien consiste à prouver qu'on ne compte parmi ses ascendants aucun juif ni musulman (ni un de leurs descendants nouvellement converti). L'histoire et les effets de ces statuts sont passionnants à étudier, mais on ne saurait ici en amorcer l'analyse. Voir R. Carrasco, L'Espagne classique 1414-1814, Paris, Hachette, 1992; I.S. Révah, «La controverse sur les statuts de pureté de sang», Revue des Études juives, LXXIII (3-4), juil.-déc. 1971; B. Bennassar (éd.), L'Inquisition espagnole XVIe-XIXe siècles, Paris, Hachette, 1978.

6. P. Chaunu, op. cit. (cf. note 3), p. 162. Les statuts de pureté de sang ne disparaîtront qu'au XIX ${ }^{e}$ siècle malgré plusieurs réelles mais vaines tentatives, faites d'ailleurs par des dignitaires de l'Église espagnole, pour en limiter les pouvoirs. Pour comprendre les bouleversements sociaux que cela provoque, on peut lire ce passage de R. Carrasco, op. cit., p. 101: «La race, c'était donc la terrible tache, ou mancha qu'il fallait à tout prix tenir secrète aux yeux de l'opinion. Ainsi, la pureté de sang, en détournant les solidarités traditionnelles à dominante hiérarchique - lignages, clans et clientèles - au profit d'un système de discrimination raciale, transforma-t-elle radicalement les rapports sociaux dans l'Espagne classique. Elle introduisait en effet une nouvelle ligne de démarcation dans le système de l'honneur entre ceux qui pouvaient se prévaloir d'une ascendance sans tache et les autres, qui se trouvaient être justement les membres de l'élite politique, économique et culturelle».

7. H. Méchoulan, Le Sang de l'autre ou l'honneur de Dieu. Indiens, juifs et morisques au Siècle d'Or, Paris, Fayard, 1979, p. 138-143.

8. Ibid., p. 10.

9. Voir entre autres B. Bennassar, Un Siècle d'Or espagnol, Paris, Laffont,1982; C. Chauchadis, Honneur, morale et société dans l'Espagne de Philippe II, Paris, Éd. du CNRS, 1984.

10. Pour l'analyse du roman picaresque, voir entre autres M. Molho, "Judaïsme, picarisme et marchandise: sur le discours de Mateo Aleman et la Vie de Guzman de Alfarache», Revue des Études juives, CXLIV (1-3), janv-sept. 1985, p. 71-80.

11. "Le Moi et le Ça», Essais de psychanalyse, Paris, Payot, 1981, p. 238. 12. M. Merleau-Ponty, Le Visible et l'Invisible, Paris, Gallimard, 1964, p. 183.

13. J. Lacan, «Le stade du miroir comme formateur de la fonction du
Je telle qu'elle nous est révélée dans l'expérience psychanalytique", Écrits, Paris, Seuil, 1966, p. 99.

14. Ibid., p. 100.

15. Folie ou psychose qu'il faut bien sûr considérer avec toutes ses contradictions dont la plus intéressante est sans doute qu'elle n'empêche pas les multiples entreprises de traductions et d'éditions critiques de la Bible de proliférer, sans compter qu'avec la fondation par Cisneros de l'université d'Alcala de Henares (ville natale de Cervantès), au lendemain de l'expulsion des juifs (l'université s'annonce dès 1495 et ouvre en 1509), se poursuit un enseignement de la science théologique et de l'humanisme incluant bien sûr celui des langues, dont l'hébreu n'est pas exclu mais au contraire incontournable. Voir R. Carrasco, op. cit.; M. Bataillon, Érasme et l'Espagne, en particulier le premier chapitre «Cisneros et la préréforme espagnole ", Genève, Droz, 1991.

16. Dette qui a été entre autres analysée par Freud dans L'Homme Moise et la religion monothéiste, Paris, Gallimard, [1939] 1986.

17. Op. cit. p. 147.

18. Même l'Église catholique et les gens d'église reconnaîtront que l'Espagne des statuts de pureté de sang a oublié la loi chrétienne de l'amour du prochain et de l'interdit du meurtre. Le dérapage imaginaire est dès lors maximal dans la mesure où il se radicalise au point de révéler encore une fois que l'imaginaire est toujours, dans son essence, imaginaire de la fin.

19. H. Méchoulan, op. cit, p. 21 et 136.

20. Le terme pila, employé au chapitre III pour nommer l'auge, se traduit aussi par vasque, bénitier, fonts baptismaux.

21. Voir à ce sujet la superbe et encore lumineuse lecture de Marthe Robert dans L'Ancien et le Nouveau, Paris, Grasset, coll. «Les cahiers rouges", 1963.

22. Par exemple, J.J. Saer, «Don Quichotte en liberté», entretien avec G. de Cortanze, Magazine littéraire: Cervantès, n 358, octobre 1997.

23. B. Gracian, Art et figures de l'esprit (Agudeza y arte del ingenio, 1647), Paris, Seuil, 1983, p. 279: «La Vérité était l'épouse légitime de l'Entendement, mais Tromperie, sa grande rivale, entreprit de lui arracher sa place dans ce lit et de la chasser de son trône. [...] La Vérité ouvrit les yeux, détermina de n'aller plus sans artifice et, depuis lors, use d'invention, s'insinue par des détours, triomphe par des stratagèmes, peint ce qui est loin comme étant près, parle du présent au passé, dénonce chez un sujet ce qu'elle veut condamner dans un autre [...] et, par d'ingénieuses circonvolutions, en vient toujours au point précis de son intention".

24. H. Méchoulan, op. cit., p. 177.

25. Même si ce corps de l'hidalgo est pour quelque chose dans l'entreprise de déconstruction de la chevalerie, on peut rappeler la misère réelle de ces nobles de l'époque par ce commentaire de $\mathrm{M}$. de Unamuno: "Misérable le noble qui, pour entretenir son honneur, fait pauvre chère à huis clos, et puis, payant de mine, sort dans la rue avec un cure-dent, bien qu'il n'ait mangé chose qui l'oblige à se les nettoyer. Misérable dis-je, celui qui a l'honneur chatouilleux et croit que l'on voit d'une lieue le rapetassement de son soulier, la graisse de son chapeau, la corde de son manteau et la faim de son estomac", cité par M.

Escamilla-Colin, Crimes et châtiments dans l'Espagne inquisitoriale, Paris, Berg International, 2 vol., 1992.

26. Éd. espagnole: M. de Cervantes, Don Quijote de la Mancha, Madrid, Editorial Everest, S.A., 1997; texto y notas, L. Casanovas Marqués; illustrado por V. Zanetti.

27. Don Quichotte II, Paris, Gallimard, coll. «Folio », p. 618.

28. Voir D. Aubier, Don Quichotte prophète d'Israël, Paris, Laffont, 1966 
et R. Reichelberg, Don Quichotte ou le roman d'un juif masqué, Paris, Entailles-Philippe Nadal, 1989.

29. Voir aussi R. Barkaï (dir.), Chrétiens, musulmans et juifs dans l'Espagne médiévale. De la convergence à l'expulsion, Paris, Cerf, 1994. Après avoir rappelé les nombreux dérivés de l'arabe présents dans la langue espagnole, l'auteur ajoute: «Mais la présence musulmane et juive dans la culture espagnole et dans l'histoire de la péninsule ibérique ne se réduit pas seulement à des signes extérieurs et linguistiques. Derrière tout cela, une question se pose, grave et difficile: celle des strates mentales déposées par les deux cultures sémitiques dans la personnalité de l'individu et de la collectivité espagnoles".

30. Voir H. Méchoulan, op. cit., p. 144 : «L'Espagnol souffrait d'une impatience de l'éternité qui déformait toute vision rationnelle de l'existence. Les succès militaires, les conversions, bref tous les procédés d'identification réussis, reconduisaient à chaque instant le contrat divin aux termes duquel l'Espagne acceptait de servir aveuglément les desseins de Dieu ".

31. Ainsi trouve-t-on dans le Dictionnaire des noms des personnages $d u$ Don Quichotte de Cervantes, de D. Reyre, Paris, Éd. hispaniques, 1980, les rubriques suivantes: "Quixada: nom sobriquet servant dans l'anthroponymie à désigner un homme à forte mâchoire"; "Quesade: gâteau à base de fromage (queso) que l'on mangeait au carnaval »; "Quixana: qui est sain; (Qui es sanus-Quisana-Quixana) nom de l'hidalgo avant qu'il soit gagné par la folie chevaleresque. Le masculin Quisano, désigne à la fin du roman le héros guéri de sa folie (Alonso Quijano el bueno)"; "Quixote (Quijote): dérivé de gigot; cuissot, pièce de l'armure; la cuisse est aussi le symbole universel de la possession érotique, nom ironique pour désigner le plus chaste des amants", etc. Toutes ces références burlesques à la fonction orale ne sont pas négligeables. Don Quichotte a dévoré les livres et ne vit que de la parole, tel un prophète d'Israël.

32. Ibid. : «ben, en arabe et en hébreu : fils de; Ingil : évangile; Éli: mon Dieu; iggel: Cerf, ce qui fait de l'historien arabe un "fils du Cerf", c'est-à-dire de Cervantes ». On peut encore ajouter angeli, pour anges, ce qui donne Seigneur Vérité, fils des anges.

33. Les nombreuses controverses entre chrétiens et juifs qui ont occupé l'Espagne d'avant l'expulsion ont toujours pour noyau la reconnaissance du Messie, dont les «signes" sont débattus en place publique, dans le but, bien sûr, de faire entendre au peuple juif son erreur. La figure de l'âne revient souvent lors de ces disputes. Voir Nahmanide, La Dispute de Barcelone, Paris, Verdier, 1984. Dans leur introduction au procès-verbal de cette dispute, É. Smilévitch et L. Ferrier écrivent: «La question du Messie est la question la plus radicale que l'on puisse poser au sujet du pouvoir; elle est celle de la continuité et de la pérennité des peuples. [...] À travers elle, c'est le sens de l'exil du peuple juif, dépossédé de la souveraineté, qui devient l'enjeu de l'affrontement. Si le Messie est déjà venu et que les Juifs ne l'ont pas connu, leur exil n'est plus qu'une inutile errance" ".

34. Car cette mise en scène d'auteurs fictifs étrangers était une loi du genre.

35. J'emprunte ce titre au premier livre de Guillaume Apollinaire qui raconte comment l'enchanteur Merlin qui était immortel, fut enchanté et tué par la Dame du Lac. Or l'enchanteur est mort mais non son âme qui continue de faire entendre sa voix d'outre-sépulcre.

36. Ainsi, toute l'histoire du crypto-christianisme de la belle Zoraïde dans la dernière partie du premier livre. Crypto-christianisme qui permet d'inverser bien sûr le marranisme espagnol, et tente de faire porter à l'un le destin de l'autre.

37. R. Israël, «Sommes-nous tous des marranes? ", Les Nouveaux Cahiers $\mathrm{n}^{\circ} 59$, hiver 1979-80, p. 27.

38. Ibid., p. 30 C'est toute la question de l'identité juive qui s'en trouve éclairée: «La judéité n'est rien d'autre que cette façon lucidement particulière d'être le semblable de l'Autre, tout en étant assez distinct pour lui renvoyer une parole qui soit vraie et non un écho [ou une pure image]".

39. À cet égard, une blague juive nous donne un peu le sens de cette «folie» catholique pour celui qui y est amené de force: «Au temps de l'Inquisition, un espion fait subitement irruption chez un homme récemment converti (de force) au christianisme. C'est un vendredi et pourtant il est à table en train de manger du poulet! - Comment, tu manges du poulet! Tu es un faux chrétien, alors! - Pas du tout, c'est du poisson! Je sais bien qu'on fait maigre le vendredi. - Tu veux me faire croire que c'est du poisson alors que je vois bien du poulet! - Mais non! J'ai fait comme vous: je l'ai aspergé d'un peu d'eau, j'ai fait le signe de la croix et j'ai dit, Ceci est du poisson, Ceci est du poisson! Et voilà! Maintenant c'est du poisson». M.-A. Ouaknin et D. Rotnemer, La Bible de l'humour juif, Paris, Ramsay, 1995.

40. "No siempre hay tocinos donde hay estacas". Autrement dit, même les crochets pour suspendre le lard deviennent un signe qui, de ce fait même, se voit frappé de suspicion.

41. "[...] ce qu'il a laissé d'écrire» est une tournure un peu archaïque pour «ce qu'il n'a pas écrit». 